\title{
ESTIMATES OF THE EXIT PROBABILITY FOR TWO CORRELATED BROWNIAN MOTIONS
}

\author{
JINGHAI SHAO* AND \\ XIUPING WANG, ${ }^{* *}$ Beijing Normal University
}

\begin{abstract}
Given two correlated Brownian motions $\left(X_{t}\right)_{t \geq 0}$ and $\left(Y_{t}\right)_{t \geq 0}$ with constant correlation coefficient, we give the upper and lower estimations of the probability $\mathbb{P}\left(\max _{0 \leq s \leq t} X_{s} \geq\right.$ $a, \max _{0 \leq s \leq t} Y_{s} \geq b$ ) for any $a, b, t>0$ through explicit formulae. Our strategy is to establish a new reflection principle for two correlated Brownian motions, which can be viewed as an extension of the reflection principle for one-dimensional Brownian motion. Moreover, we also consider the nonexit probability for linear boundaries, i.e. $\mathbb{P}\left(X_{t} \leq\right.$ $\left.a t+c, Y_{t} \leq b t+d, 0 \leq t \leq T\right)$ for any constants $a, b \geq 0$ and $c, d, T>0$.
\end{abstract}

Keywords: Boundary crossing probability; correlated Brownian motion; exit probability; reflection principle

2010 Mathematics Subject Classification: Primary 60J65

Secondary $60 \mathrm{G} 40$

\section{Introduction}

Boundaries crossing probabilities or exit probabilities of Brownian motion have many applications in nonparametric statistics, sequential analysis, and change point problems in econometrics, biology, epidemiology, and mathematical finance. Let $(\Omega, \mathbb{P}, \mathcal{F})$ be a given probability space with a reference family $\left(\mathcal{F}_{t}\right)_{t \in[0, \infty)}$. A one-dimensional continuous process $\left(X_{t}\right)_{t \geq 0}$ is called a one-dimensional Brownian motion if it is $\mathcal{F}_{t}$-adapted with $X_{0}=0$ and satisfies

$\mathbb{E}\left[\exp \left[\mathrm{i} \lambda\left(X_{t}-X_{s}\right)\right] \mid \mathcal{F}_{s}\right]=\exp \left[-\frac{1}{2}(t-s) \lambda^{2}\right], \quad$ almost surely, for every $\lambda \in \mathbb{R}, 0<s<t$.

It is well known that the Brownian motion admits the reflection principle, that is, for each given stopping time $\tau$, define a new process

$$
\tilde{X}_{t}=2 X_{t \wedge \tau}-X_{t}
$$

where $t \wedge \tau=\min \{t, \tau\}$; then the process $\left(\tilde{X}_{t}\right)_{t \geq 0}$ is a new Brownian motion. This property plays a very important role in estimating the exit probabilities of Brownian motion and stochastic processes. Applying this property to the stopping time $\tau=\tau_{x}^{a}:=\inf \left\{t>0, X_{t} \geq a\right\}$, we can obtain the distribution of $X^{*}(t)=X_{t}^{*}:=\max _{0 \leq s \leq t} X_{s}$, given by

$$
\mathbb{P}\left(X^{*}(t) \geq a\right)=\mathbb{P}\left(\tau_{x}^{a} \leq t\right)=\mathbb{P}\left(\left|X_{t}\right| \geq a\right) \text { for every } a>0 .
$$

Received 26 February 2010; revision received 21 April 2012.

* Postal address: School of Mathematical Sciences, Beijing Normal University, Beijing 100875, China.

Email address: shaojh@bnu.edu.cn

** Postal address: Beijing Normal University, Xin Jie Kou Wai Da Jie 19, Beijing 100875, China.

Email address: wangxp@yahoo.com 
Based on this fundamental fact, there are many results on the estimates of the exit probability for one-dimensional Brownian motion with respect to a moving boundary, that is, for a nonrandom positive continuous function $f(t)$, estimate the probability

$$
\mathbb{P}\left(X_{t} \leq f(t), 0 \leq t \leq T\right) \text { for some finite or infinite constant } T>0 .
$$

For example, Doob [2] obtained an explicit formula for the following linear boundary crossing probability:

$$
\mathbb{P}\left(X_{t} \leq a t+b, t \geq 0\right)=1-\mathrm{e}^{-2 a b} \quad \text { for every } a, b>0 .
$$

However, explicit formulae have been obtained only for linear and a few special boundaries $f(t)$; for instance, Anderson [1] dealt with linear upper and lower boundaries. For more general boundaries and a higher-dimensional Brownian motion, most methods seek to give approximations or estimates of the corresponding probability. We will not mention too much on this intensively studied topic, but refer the reader to [3], [9]-[11], and the references therein for various methods (including solving differential or integral equations, Monte Carlo path simulation, and series expansion) to deal with different boundaries.

In this paper we focus on the joint distribution of the exit probability of two correlated Brownian motions. Specifically, let $\left(X_{t}\right)_{t \geq 0}$ and $\left(Y_{t}\right)_{t \geq 0}$ be two one-dimensional $\mathcal{F}_{t}$-Brownian motions, which are not necessary mutually independent, but their joint distribution is normal. Let

$$
X^{*}(t)=X_{t}^{*}=\max _{0 \leq s \leq t} X_{s}, \quad Y^{*}(t)=Y_{t}^{*}=\max _{0 \leq s \leq t} Y_{s} .
$$

For any positive constants $a$ and $b$, consider the probability

$$
\mathbb{P}\left(X^{*}(t) \geq a, Y^{*}(t) \geq b\right) .
$$

This probability depends strongly on the dependence between the processes $\left(X_{t}\right)_{t \geq 0}$ and $\left(Y_{t}\right)_{t \geq 0}$. For instance, assume that $\mathbb{P}\left(X^{*}(t) \geq a\right)=\mathbb{P}\left(Y^{*}(t) \geq a\right)=0.1$. If the two processes are independent then

$$
\mathbb{P}\left(X^{*}(t) \geq a, Y^{*}(t) \geq a\right)=\mathbb{P}\left(X^{*}(t) \geq a\right)^{2}=0.01 .
$$

But, if $X_{t}=Y_{t}$ for every $t \geq 0$ then

$$
\mathbb{P}\left(X^{*}(t) \geq a, Y^{*}(t) \geq a\right)=\mathbb{P}\left(X^{*}(t) \geq a\right)=0.1 .
$$

Therefore, the mutual dependence of two stochastic processes plays a very important role in the calculation of the exit probability. In applications, neglecting the dependence of two stochastic processes may cause serious errors in the estimation of the exit probability, which may represent the risk of bankruptcy for a company or the risk of breakdown for a system.

We now introduce the basic assumptions used in this paper. Noting that $X_{0}=Y_{0}=0$, set $r(t)$ to be the correlation coefficient between $X_{t}$ and $Y_{t}$ :

$$
r(t):=\frac{\mathbb{E}\left[X_{t} Y_{t}\right]}{\sqrt{\mathbb{E} X_{t}^{2} \mathbb{E} Y_{t}^{2}}}, \quad t>0 .
$$

Throughout this paper, we assume that the following hypotheses hold.

(H1) For every $t>0$, the correlation coefficient $r(t)$ is equal to a constant $r$.

(H2) For any $t>s \geq 0,\left(X_{t}-X_{s}, Y_{t}-Y_{s}\right)$ admits a normal distribution as well. 
Before introducing our results, let us review some known results on this kind of problem. Assume that hypotheses (H1) and (H2) hold, and that $r^{2} \neq 1$. For any given positive constants $a$ and $b$, according to the works [6] and [7], it holds that

$$
\begin{aligned}
& \mathbb{P}\left(X^{*}(t)<a, Y^{*}(t)<b\right) \\
& \quad=\frac{2 r_{0}}{\sqrt{2 \pi t}} \mathrm{e}^{-r_{0}^{2} / 4 t} \sum_{n \text { odd }} \frac{1}{n} \sin \left(\frac{n \pi \theta_{0}}{\alpha}\right)\left[I_{\left(v_{n}-1\right) / 2}\left(\frac{r_{0}^{2}}{4 t}\right)+I_{\left(v_{n}+1\right) / 2}\left(\frac{r_{0}^{2}}{4 t}\right)\right],
\end{aligned}
$$

where $\left(r_{0}, \theta_{0}\right)$ denotes the radial coordinate and angular coordinate of the point $((a-$ $\left.r b) / \sqrt{1-r^{2}}, b\right), v_{n}=n \pi / \alpha$,

$$
\alpha= \begin{cases}\pi+\tan ^{-1}\left(-\frac{\sqrt{1-r^{2}}}{r}\right), & r>0, \\ \frac{1}{2} \pi, & r=0, \\ \tan ^{-1}\left(-\frac{\sqrt{1-r^{2}}}{r}\right), & r<0,\end{cases}
$$

and $I_{v}$ denotes the modified Bessel function of the first kind of order $v$, which can be expressed by

$$
I_{v}(x)=\sum_{m=0}^{\infty} \frac{1}{m ! \Gamma(m+v+1)}\left(\frac{x}{2}\right)^{2 m+v}
$$

with $\Gamma(z)$ the gamma function. This explicit formula is not as explicit as one might desire for practical applications and numerical computations, and its use makes it difficult to progress further theoretically. For instance, as pointed out in [7], using (1.3) to approximate the exit probability of a time-dependent boundary is computationally expensive to the point of being prohibitive. Moreover, the method of obtaining the above result depends on the explicit solution of the heat equation in a wedge. Therefore, it is difficult to extend to the higher-dimensional case.

The following results will provide an easily computed estimate of the desired exit probability. Our strategy to estimate (1.2) is to establish a new type of reflection principle for two correlated Brownian motions (stated in Section 2), which is an extension of the reflection principle for a one-dimensional Brownian motion.

The following theorem is our first main result.

Theorem 1.1. Let $\left(X_{t}\right)_{t>0}$ and $\left(Y_{t}\right)_{t \geq 0}$ be two $\mathcal{F}_{t}$-Brownian motions having normal joint distribution. Assume that hypotheses (H1) and (H2) hold, and that $r^{2} \neq 1$. Then, for any positive constants $a$ and $b$,

$$
\mathbb{P}\left(X^{*}(t) \geq a, Y^{*}(t) \geq b\right) \leq \min \left\{U_{1}(a, b), U_{2}(a, b)\right\}
$$

and

$$
\mathbb{P}\left(X^{*}(t) \geq a, Y^{*}(t) \geq b\right) \geq \max \left\{U_{1}(a, b)-\mathcal{T}_{1}(a, b), U_{2}(a, b)-\mathcal{T}_{2}(a, b)\right\},
$$

where

$$
\Phi(z)=\int_{-\infty}^{z} \frac{1}{\sqrt{2 \pi}} \mathrm{e}^{-x^{2} / 2} \mathrm{~d} x, \quad \varphi(x, y)=\frac{1}{2 \pi \sqrt{1-r^{2}}} \exp \left[-\frac{x^{2}-2 r x y+y^{2}}{2\left(1-r^{2}\right)}\right],
$$


and

$$
\begin{aligned}
& U_{1}(a, b)=\Phi\left(\frac{-b}{\sqrt{t}}\right)+\int_{-\infty}^{-a / \sqrt{t}}\left(\int_{(b-2 r a) / \sqrt{t}}^{\infty} \varphi(x, y) \mathrm{d} y\right) \mathrm{d} x \\
& +\int_{a / \sqrt{t}}^{\infty}\left(\int_{b / \sqrt{t}}^{\infty} \varphi(x, y) \mathrm{d} y\right) \mathrm{d} x \\
& U_{2}(a, b)=\Phi\left(\frac{-a}{\sqrt{t}}\right)+\int_{(a-2 r b) / \sqrt{t}}^{\infty}\left(\int_{-\infty}^{-b / \sqrt{t}} \varphi(x, y) \mathrm{d} y\right) \mathrm{d} x \\
& +\int_{a / \sqrt{t}}^{\infty}\left(\int_{b / \sqrt{t}}^{\infty} \varphi(x, y) \mathrm{d} y\right) \mathrm{d} x \text {, } \\
& \mathcal{T}_{1}(a, b)=\int_{0}^{t}\left\{\int _ { - \infty } ^ { a } \left[\int_{-\infty}^{(a-x) / \sqrt{t-u}}\left(\int_{-\infty}^{0} \varphi(z, w) \mathrm{d} w\right) \mathrm{d} z\right.\right. \\
& \left.-\int_{-\infty}^{(x-a) / \sqrt{t-u}}\left(\int_{-\infty}^{2 r(x-a) / \sqrt{t-u}} \varphi(z, w) \mathrm{d} w\right) \mathrm{d} z\right] \\
& \left.\times \frac{\mathrm{e}^{-(x-r b)^{2} / 2 u\left(1-r^{2}\right)}}{\sqrt{2 \pi u\left(1-r^{2}\right)}} \mathrm{d} x\right\} \frac{b}{\sqrt{2 \pi u^{3}}} \mathrm{e}^{-b^{2} / 2 u} \mathrm{~d} u, \\
& \mathcal{T}_{2}(a, b)=\int_{0}^{t}\left\{\int _ { - \infty } ^ { b } \left[\int_{-\infty}^{(b-x) / \sqrt{t-u}}\left(\int_{-\infty}^{0} \varphi(z, w) \mathrm{d} z\right) \mathrm{d} w\right.\right. \\
& \left.-\int_{-\infty}^{(x-b) / \sqrt{t-u}}\left(\int_{-\infty}^{2 r(x-b) / \sqrt{t-u}} \varphi(z, w) \mathrm{d} z\right) \mathrm{d} w\right] \\
& \left.\times \frac{\mathrm{e}^{-(x-r a)^{2} / 2 u\left(1-r^{2}\right)}}{\sqrt{2 \pi u\left(1-r^{2}\right)}} \mathrm{d} x\right\} \frac{a}{\sqrt{2 \pi u^{3}}} \mathrm{e}^{-a^{2} / 2 u} \mathrm{~d} u .
\end{aligned}
$$

Remark 1.1. Theorem 1.1 provides lower and upper estimates of the exit probability for two correlated Brownian motions with constant correlation coefficient $r$ varying in the interval $(-1,1)$. For the case in which $r=1$, which means that $X_{t}=Y_{t}$ almost surely, the problem in two-dimensional space is reduced to that in one-dimensional space and the exit probability is known. For the case in which $r=-1$, which means that $Y_{t}=-X_{t}$ almost surely, it is known that

$$
\mathbb{P}\left(\left|X_{s}\right| \leq a, 0 \leq s \leq t\right)=\frac{4}{\pi} \sum_{n=0}^{\infty} \frac{(-1)^{n}}{2 n+1} \exp \left[-\frac{(2 n+1)^{2} \pi^{2} t}{8 a^{2}}\right]
$$

(see, for example, [4, Chapter X, Equation (5.9)]).

Remark 1.2. To see the usefulness of our estimates, it is easy to check that

$$
\mathcal{T}_{1}(a, b)=O\left(t^{-1 / 2}\right), \quad \mathcal{T}_{2}(a, b)=O\left(t^{-1 / 2}\right), \quad \text { as } t \rightarrow \infty,
$$

and, for all $p>0$,

$$
\lim _{b \rightarrow \infty} b^{p} \widetilde{\mathcal{T}}_{1}(a, b)=0, \quad \lim _{a \rightarrow \infty} a^{p} \widetilde{\mathcal{T}}_{2}(a, b)=0 .
$$

So the difference between the upper and lower bounds given in Theorem 1.1 is as small as $t, a$, or $b$ is large. 
As an application of Theorem 1.1, let us return to the study of the following quantity again:

$$
H(t, b ; r):=\frac{\mathbb{P}\left(X_{t}^{*} \geq b, Y_{t}^{*} \geq b\right)}{\mathbb{P}\left(Y_{t}^{*} \geq b\right)} .
$$

As mentioned above, for each $t>0$, if $r=0$ then $\lim _{b \rightarrow \infty} H(t, b ; r)=0$ and if $r=1$ then $\lim _{b \rightarrow \infty} H(t, b ; r)=1$. For $r \in(-1,1)$, we will show that $\lim _{\sup _{b \rightarrow \infty}} H(t, b ; r) \leq \frac{1}{2}$ for each $t>0$. This is an interesting phenomenon, since it means that, when $b$ is large enough, the quantity

$$
\frac{\mathbb{P}\left(X_{t}^{*} \geq b, Y_{t}^{*} \geq b\right)}{\mathbb{P}\left(Y_{t}^{*} \geq b\right)}
$$

is not a continuous function of the correlation coefficient $r$ and is bounded above by a constant $\frac{1}{2}$.

Corollary 1.1. It holds for $r \in(-1,1)$ and each $t>0$ that

$$
\limsup _{b \rightarrow \infty} \frac{\mathbb{P}\left(X_{t}^{*} \geq b, Y_{t}^{*} \geq b\right)}{\mathbb{P}\left(Y_{t}^{*} \geq b\right)} \leq \frac{1}{2} .
$$

The calculation of the exit probability for a stochastic process relative to a general boundary is also very important in applications. However, usually, it is even more difficult to obtain an explicit formula. As pointed out in [11] and [12], the exit probability of a general boundary for one-dimensional Brownian motion can be approximated by the exit probability of a linear boundary. Therefore, it is of great importance to calculate the exit probability of a linear boundary. Here, based on Theorem 1.1, and using Girsanov's theorem, we can obtain lower and upper estimates of the crossing probability of the process $\left(X_{t}, Y_{t}\right)_{t \geq 0}$ for linear moving boundaries.

Theorem 1.2. Let $\left(X_{t}\right)_{t \geq 0}$ and $\left(Y_{t}\right)_{t \geq 0}$ be two $\mathcal{F}_{t}$-Brownian motions having normal joint distribution. Assume that hypotheses (H1) and (H2) hold, and that $|r|<1$. Let $a, b \geq 0$ and $c, d>0$. Then, for $T>0$ and $p>1$,

$$
\mathbb{P}\left(X_{t} \leq a t+c, Y_{t} \leq b t+d, 0 \leq t \leq T\right) \leq A_{p} \mathbb{P}\left(X^{*}(T) \leq c, Y^{*}(T) \leq d\right)^{1 / p}
$$

and

$$
\mathbb{P}\left(X_{t} \leq a t+c, Y_{t} \leq b t+d, 0 \leq t \leq T\right) \geq B_{p} \mathbb{P}\left(X^{*}(T) \leq c, Y^{*}(T) \leq d\right)^{p},
$$

where the constants

$$
A_{p}=\left(\mathbb{E} \tilde{M}_{T}^{p /(p-1)}\right)^{(p-1) / p}<+\infty, \quad B_{p}=\left(\mathbb{E} M_{T}^{p /(p-1)}\right)^{1-p}>0 .
$$

Here the random variables $\tilde{M}_{T}$ and $M_{T}$ are defined by

$$
\begin{aligned}
& \tilde{M}_{T}=\exp \left[\frac{-1}{2\left(1-r^{2}\right)}\left\{2(a-r b) X(T)+2(b-r a) Y(T)+\left(a^{2}+b^{2}-2 r a b\right) T\right\}\right], \\
& M_{T}=\exp \left[\frac{1}{2\left(1-r^{2}\right)}\left\{2(a-r b) X(T)+2(b-r a) Y(T)-\left(a^{2}+b^{2}-2 r a b\right) T\right\}\right] .
\end{aligned}
$$

Remark 1.3. 1. Since the random variables $X^{*}(T)$ and $Y^{*}(T)$ have the same distribution as $|X(T)|$ and $|Y(T)|$, respectively, combining this fact with Theorem 1.1, we know that 
the probability $\mathbb{P}\left(X^{*}(T) \leq c, Y^{*}(T) \leq d\right)$ can be estimated through an explicit formula. Therefore, Theorem 1.2 gives explicit lower and upper bounds for the crossing probability of linear boundaries.

2. In Theorem 1.2, we assume that $|r|<1$. For $r=1$ or -1 , this yields $Y_{t}=X_{t}$ or $Y_{t}=-X_{t}$ a.s. for every $t>0$. Then the problem reduces to the case of one-dimensional Brownian motion; see [11] and [12] for results in this case.

In Section 2 we provide the proofs of Theorem 1.1 and Corollary 1.1, and also give the reflection principle for two correlated Brownian motions. In Section 3 we provide the proof of Theorem 1.2.

\section{Proofs of Theorem 1.1 and Corollary 1.1}

The idea of the proof of Theorem 1.1 is to study the reflection property of the two-dimensional process $\left(X_{t}, Y_{t}\right)_{t \geq 0}$, which is stated in Lemma 2.2 below. This will be a natural extension of the reflection principle for one-dimensional Brownian motion.

Lemma 2.1. Let $\left(X_{t}\right)_{t \geq 0}$ and $\left(Y_{t}\right)_{t \geq 0}$ be two $\mathcal{F}_{t}$-Brownian motions with normal joint distribution. Assume that hypotheses $(H 1)$ and $(H 2)$ hold. Then the process $\left(X_{t}, Y_{t}\right)_{t \geq 0}$ has the same distribution as that of the process $\left(X_{t}-2 r Y_{t},-Y_{t}\right)_{t \geq 0}$.

Proof. It is easy to see that $t \mapsto X_{t}-2 r Y_{t}$ is an $\mathcal{F}_{t}$-martingale. For $0<s<t$,

$$
\begin{aligned}
& \mathbb{E}\left[\left(X_{t}-2 r Y_{t}\right)^{2} \mid \mathcal{F}_{s}\right]-\left(X_{s}-2 r Y_{s}\right)^{2} \\
& \quad=\mathbb{E}\left[\left(X_{t}-X_{s}\right)^{2}-4 r\left(X_{t} Y_{t}-X_{s} Y_{s}\right)+4 r^{2}\left(Y_{t}-Y_{s}\right)^{2} \mid \mathcal{F}_{s}\right] \\
& \quad=t-s .
\end{aligned}
$$

Then $t \mapsto\left(X_{t}-2 r Y_{t}\right)^{2}-t$ is also an $\mathcal{F}_{t}$-martingale. By the Lévy characterization theorem of Brownian motion, the process $\left(X_{t}-2 r Y_{t}\right)_{t \geq 0}$ is a new Brownian motion. Furthermore, for every $t>0$,

$$
\frac{\mathbb{E}\left(X_{t}-2 r Y_{t}\right)\left(-Y_{t}\right)}{\sqrt{\mathbb{E}\left(X_{t}-2 r Y_{t}\right)^{2} \mathbb{E}\left(-Y_{t}\right)^{2}}}=r .
$$

Therefore, the process $\left(X_{t}-2 r Y_{t},-Y_{t}\right)_{t \geq 0}$ has the same distribution as that of the process $\left(X_{t}, Y_{t}\right)_{t \geq 0}$.

Lemma 2.2. (Reflection principle: first type.) Let $\tau$ be a stopping time with respect to $\left(\mathcal{F}_{t}\right)_{t \geq 0}$. Let

$$
\begin{aligned}
\tilde{X}_{t} & =X_{t \wedge \tau}+\left(X_{t}-X_{t \wedge \tau}-2 r\left(Y_{t}-Y_{t \wedge \tau}\right)\right) \mathbf{1}_{\{t>\tau\}}, \\
\tilde{Y}_{t} & =Y_{t \wedge \tau}+\left(Y_{t \wedge \tau}-Y_{t}\right) \mathbf{1}_{\{t>\tau\}},
\end{aligned}
$$

where $t \wedge \tau=\min \{t, \tau\}$. Then the processes $\left(\tilde{X}_{t}, \tilde{Y}_{t}, \tau\right)_{t \geq 0}$ and $\left(X_{t}, Y_{t}, \tau\right)_{t \geq 0}$ admit the same distribution.

Proof. Let $\bar{Y}_{s}=Y_{s+\tau}-Y_{\tau}$ and $\bar{X}_{s}=X_{s+\tau}-X_{\tau}$ for every $s \geq 0$. Then we have

$$
X_{t}=X_{t \wedge \tau}+\bar{X}_{t-\tau} \mathbf{1}_{\{t>\tau\}}, \quad Y_{t}=Y_{t \wedge \tau}+\bar{Y}_{t-\tau} \mathbf{1}_{\{t>\tau\}},
$$

and

$$
\tilde{Y}_{t}=Y_{t \wedge \tau}-\bar{Y}_{t-\tau} \mathbf{1}_{\{t>\tau\}}, \quad \tilde{X}_{t}=X_{t \wedge \tau}+\left(\bar{X}_{t-\tau}-2 r \bar{Y}_{t-\tau}\right) \mathbf{1}_{\{t>\tau\}} .
$$


By the strong Markov property of Brownian motion, $\bar{X}_{S}$ and $\bar{Y}_{S}$ are independent of the $\sigma$-algebra $\widetilde{F}_{\tau}$. Furthermore, it follows from Lemma 2.1 that $\left(\bar{X}_{t-\tau}, \bar{Y}_{t-\tau}\right)$ has the same distribution as the process $\left(\bar{X}_{t-\tau}-\underset{\widetilde{X}}{2} r \bar{Y}_{t-\tau},-\bar{Y}_{t-\tau}\right)$. Then the process $\left(X_{t}, Y_{t}, \tau\right)_{t \geq 0}$ has the same distribution as the process $\left(\widetilde{X}_{t}, \widetilde{Y}_{t}, \tau\right)_{t \geq 0}$.

The idea of Lemma 2.2 is that when making a reflection for the process $\left(Y_{t}\right)_{t \geq 0}$ at the stopping time $\tau$, we make a necessary modification in the process $\left(X_{t}\right)_{t \geq 0}$ to guarantee that the new generated process admits the same distribution as the process $\left(X_{t}, Y_{t}\right)_{t \geq 0}$ after the stopping time $\tau$. Of course, we can interchange $\left(X_{t}\right)_{t \geq 0}$ and $\left(Y_{t}\right)_{t \geq 0}$ to make a reflection for the process $\left(X_{t}\right)_{t \geq 0}$ after $\tau$ and do an analogous modification for the process $\left(Y_{t}\right)_{t \geq 0}$. Both of these kinds of reflection will be used in the following argument of Theorem 1.1, depending on the choice of stopping time $\tau$.

Now we state another type of reflection principle. We will not use it in this paper, but it is of independent interest.

Lemma 2.3. (Reflection principle: second type.) Let $\tau$ be a stopping time with respect to $\left(\widetilde{F}_{t}\right)_{t \geq 0}$. Let

$$
X_{t}^{\prime}=2 X_{t \wedge \tau}-X_{t}, \quad Y_{t}^{\prime}=2 Y_{t \wedge \tau}-Y_{t}
$$

Then the process $\left(X_{t}^{\prime}, Y_{t}^{\prime}, \tau\right)_{t>0}$ admits the same distribution as the process $\left(X_{t}, Y_{t}, \tau\right)_{t>0}$.

Proof. Since

$$
X_{t}^{\prime}=X_{t \wedge \tau}-\bar{X}_{t-\tau} \mathbf{1}_{\{t \geq \tau\}}, \quad Y_{t}^{\prime}=Y_{t \wedge \tau}-\bar{Y}_{t-\tau} \mathbf{1}_{\{t \geq \tau\}},
$$

where $\bar{X}_{u}$ and $\bar{Y}_{u}$ are defined in Lemma 2.2, by virtue of arguments similar to those used in the proof of Lemma 2.2, we can obtain the desired result.

Proof of Theorem 1.1. For $a, b>0$, define the two stopping times $\tau_{x}^{a}=\inf \left\{t>0: X_{t}=a\right\}$ and $\tau_{y}^{b}=\inf \left\{t>0: Y_{t}=b\right\}$. Then $\left\{\omega \in \Omega: X^{*}(t)(\omega) \geq a\right\}=\left\{\omega \in \Omega: \tau_{x}^{a}(\omega) \leq t\right\}$ and $\left\{\omega \in \Omega: Y^{*}(t)(\omega) \geq b\right\}=\left\{\omega \in \Omega: \tau_{y}^{b}(\omega) \leq t\right\}$. We have

$$
\begin{aligned}
\mathbb{P}\left(X^{*}(t)\right. & \left.\geq a, Y^{*}(t) \geq b\right) \\
= & \mathbb{P}\left(X^{*}(t) \geq a, X_{t} \leq a, Y^{*}(t) \geq b, Y_{t} \leq b\right)+\mathbb{P}\left(X^{*}(t) \geq a, X_{t} \leq a, Y_{t} \geq b\right) \\
& +\mathbb{P}\left(X_{t} \geq a, Y^{*}(t) \geq b, Y_{t} \leq b\right)+\mathbb{P}\left(X_{t} \geq a, Y_{t} \geq b\right) \\
= & I+I I+I I I+I V .
\end{aligned}
$$

We first use Lemma 2.2 to calculate $I I$ and $I I I$. For $I I$, we use Lemma 2.2 with $X_{t}$ and $Y_{t}$ interchanged and choose $\tau$ to be the stopping time $\tau_{x}^{a}$. Thus,

$$
\begin{aligned}
I I & =\mathbb{P}\left(\tau_{x}^{a} \leq t, X_{t} \leq a, Y_{t} \geq b\right) \\
& =\mathbb{P}\left(\tau_{x}^{a} \leq t, 2 X_{\tau_{x}^{a}}-X_{t} \leq a, Y_{t}-2 r\left(X_{t}-X_{\tau_{x}^{a}}\right) \geq b\right) \\
& =\mathbb{P}\left(\tau_{x}^{a} \leq t, X_{t} \geq a, Y_{t}-2 r X_{t} \geq b-2 r a\right) \\
& =\mathbb{P}\left(X_{t} \geq a, Y_{t}-2 r X_{t} \geq b-2 r a\right) \\
& =\mathbb{P}\left(X_{t} \leq-a, Y_{t} \geq b-2 r a\right),
\end{aligned}
$$

where in the last step we used Lemma 2.1, interchanging $X_{t}$ and $Y_{t}$. For $I I I$, we use Lemma 2.2 
with $\tau=\tau_{y}^{b}$, to obtain

$$
\begin{aligned}
I I I & =\mathbb{P}\left(\tau_{y}^{b} \leq t, X_{t} \geq a, Y_{t} \leq b\right) \\
& =\mathbb{P}\left(X_{t}-2 r Y_{t}+2 r Y_{\tau_{y}^{b}} \geq a, Y_{t} \geq b\right) \\
& =\mathbb{P}\left(X_{t}-2 r Y_{t} \geq a-2 r b, Y_{t} \geq b\right) \\
& =\mathbb{P}\left(X_{t} \geq a-2 r b, Y_{t} \leq-b\right),
\end{aligned}
$$

where in the last step we used Lemma 2.1.

It now remains to only estimate $I$ :

$$
\begin{aligned}
I & =\mathbb{P}\left(\tau_{x}^{a} \leq t, X_{t} \leq a, \tau_{y}^{b} \leq t, Y_{t} \leq b\right) \\
& =\mathbb{P}\left(\tau_{y}^{b} \leq t, Y_{t} \leq b, X_{t} \leq a\right)-\mathbb{P}\left(\tau_{x}^{a} \geq t, \tau_{y}^{b} \leq t, Y_{t} \leq b\right) \\
& =\mathbb{P}\left(\tau_{y}^{b} \leq t, Y_{t} \leq b, X_{t} \leq a\right)-\mathbb{P}\left(X_{s} \leq a, 0 \leq s \leq t, \tau_{y}^{b} \leq t, Y_{t} \leq b\right) \\
& =\mathbb{P}\left(X_{t}-2 r Y_{t} \leq a-2 r b, Y_{t} \geq b\right)-\mathbb{P}\left(\tau_{x}^{a}>t, \tau_{y}^{b} \leq t, Y_{t} \leq b\right) .
\end{aligned}
$$

Applying Lemma 2.4 below, we have

$$
\begin{aligned}
0 \leq \mathbb{P}\left(X_{t}-2 r Y_{t} \leq a-2 r b, Y_{t} \geq b\right)-I \\
\leq \int_{0}^{t}\left\{\int _ { - \infty } ^ { a } \left[\int_{-\infty}^{(a-x) / \sqrt{t-u}}\left(\int_{-\infty}^{0} \varphi(z, w) \mathrm{d} w\right) \mathrm{d} z\right.\right. \\
\left.\quad-\int_{-\infty}^{(x-a) / \sqrt{t-u}}\left(\int_{-\infty}^{2 r(x-a) / \sqrt{t-u}} \varphi(z, w) \mathrm{d} w\right) \mathrm{d} z\right] \\
\left.\quad \times \frac{\mathrm{e}^{-(x-r b)^{2} / 2 u\left(1-r^{2}\right)}}{\sqrt{2 \pi u\left(1-r^{2}\right)}} \mathrm{d} x\right\} \frac{b}{\sqrt{2 \pi u^{3}}} \mathrm{e}^{-b^{2} / 2 u} \mathrm{~d} u \\
=\mathcal{T}_{1}(a, b) .
\end{aligned}
$$

Since $X_{t}$ and $Y_{t}$ play the same role in the calculation of $I$, by an analogous calculation we obtain

$$
I=\mathbb{P}\left(X_{t} \geq a, Y_{t}-2 r X_{t} \leq b-2 r a\right)-\mathbb{P}\left(\tau_{x}^{a} \leq t, X_{t} \leq a, \tau_{y}^{b}>t\right)
$$

and

$$
\begin{aligned}
0 \leq \mathbb{P}\left(X_{t}-2 r Y_{t} \leq a-2 r b, Y_{t} \geq b\right)-I \\
\leq \int_{0}^{t}\left\{\int _ { - \infty } ^ { b } \left[\int_{-\infty}^{(b-x) / \sqrt{t-u}}\left(\int_{-\infty}^{0} \varphi(z, w) \mathrm{d} z\right) \mathrm{d} w\right.\right. \\
\left.\quad-\int_{-\infty}^{(x-b) / \sqrt{t-u}}\left(\int_{-\infty}^{2 r(x-b) / \sqrt{t-u}} \varphi(z, w) \mathrm{d} z\right) \mathrm{d} w\right] \\
\left.\quad \times \frac{\mathrm{e}^{-(x-r a)^{2} / 2 u\left(1-r^{2}\right)}}{\sqrt{2 \pi u\left(1-r^{2}\right)}} \mathrm{d} x\right\} \frac{a}{\sqrt{2 \pi u^{3}}} \mathrm{e}^{-a^{2} / 2 u} \mathrm{~d} u \\
=\mathcal{T}_{2}(a, b) .
\end{aligned}
$$


Invoking the calculations for $I I$ and $I I I$, by inequality (2.1) we obtain

$$
\begin{aligned}
\mathbb{P}\left(X^{*}(t)\right. & \left.\geq a, Y^{*}(t) \geq b\right) \\
\leq & \mathbb{P}\left(X_{t} \leq a-2 r b, Y_{t} \leq-b\right)+\mathbb{P}\left(X_{t} \leq-a, Y_{t} \geq b-2 r a\right) \\
& +\mathbb{P}\left(X_{t} \geq a-2 r b, Y_{t} \leq-b\right)+\mathbb{P}\left(X_{t} \geq a, Y_{t} \geq b\right) \\
= & \mathbb{P}\left(Y_{t} \leq-b\right)+\mathbb{P}\left(X_{t} \leq-a, Y_{t} \geq b-2 r a\right)+\mathbb{P}\left(X_{t} \geq a, Y_{t} \geq b\right) \\
= & U_{1}(a, b) .
\end{aligned}
$$

If we use inequality (2.2) in the above calculation, we obtain

$$
\begin{aligned}
& \mathbb{P}\left(X^{*}(t) \geq a, Y^{*}(t) \geq b\right) \\
& \quad \leq \mathbb{P}\left(X_{t} \leq-a\right)+\mathbb{P}\left(X_{t} \geq a-2 r b, Y_{t} \leq-b\right)+\mathbb{P}\left(X_{t} \geq a, Y_{t} \geq b\right) \\
& \quad=U_{2}(a, b) .
\end{aligned}
$$

Therefore, we can obtain the following upper bound:

$$
\mathbb{P}\left(X^{*}(t) \geq a, Y^{*}(t) \geq b\right) \leq \min \left\{U_{1}(a, b), U_{2}(a, b)\right\} .
$$

Similarly, by (2.1) and (2.2) we obtain the following lower bound:

$$
\mathbb{P}\left(X^{*}(t) \geq a, Y^{*}(t) \geq b\right) \geq \max \left\{U_{1}(a, b)-\mathcal{T}_{1}(a, b), U_{2}(a, b)-\mathcal{T}_{2}(a, b)\right\} .
$$

Finally, expressing $U_{1}(a, b), U_{2}(a, b), \mathcal{T}_{1}(a, b)$, and $\mathcal{T}_{2}(a, b)$ in terms of the joint distribution function of $\left(X_{t}, Y_{t}\right)$, we can obtain (1.5) and (1.6), completing the proof of Theorem 1.1.

Lemma 2.4. For any positive $a, b, \tau_{x}^{a}$, and $\tau_{y}^{b}$ defined as above, we have

$$
\begin{aligned}
& \mathbb{P}\left(\tau_{x}^{a}>t, \tau_{y}^{b} \leq t, Y_{t} \leq b\right) \\
& \leq \int_{0}^{t}\left(\int _ { - \infty } ^ { a } \left[\int_{-\infty}^{(a-x) / \sqrt{t-u}}\left(\int_{-\infty}^{0} \varphi(z, w) \mathrm{d} w\right) \mathrm{d} z\right.\right. \\
&\left.-\int_{-\infty}^{(x-a) / \sqrt{t-u}}\left(\int_{-\infty}^{2 r(a-x) / \sqrt{t-u}} \varphi(z, w) \mathrm{d} w\right) \mathrm{d} z\right] \\
&\left.\times \frac{1}{\sqrt{2 \pi u\left(1-r^{2}\right)}} \mathrm{e}^{-(x-r b)^{2} / 2 u\left(1-r^{2}\right)} \mathrm{d} x\right) \frac{b}{\sqrt{2 \pi u^{3}}} \mathrm{e}^{-b^{2} / 2 u} \mathrm{~d} u .
\end{aligned}
$$

Proof. According to the strong Markov property of $\left(X_{t}, Y_{t}\right)_{t>0}$, we obtain

$$
\begin{aligned}
& \mathbb{P}\left(\tau_{x}^{a}>t, \tau_{y}^{b} \leq t, Y_{t} \leq b\right) \\
& =\mathbb{P}\left(X_{s}<a, 0 \leq s \leq t, \tau_{y}^{b} \leq t, Y_{t} \leq b\right) \\
& =\mathbb{P}\left(\tilde{X}_{s}<a, 0 \leq s \leq t, \tau_{y}^{b} \leq t, \tilde{Y}_{t} \leq b\right) \\
& =\mathbb{P}\left(X_{s}<a, 0 \leq s \leq \tau_{y}^{b}, X_{s}-2 r Y_{s} \leq a-2 r b, \tau_{y}^{b} \leq s \leq t, Y_{t} \geq b\right) \\
& =\mathbb{P}\left(X_{s \wedge \tau_{y}^{b}}<a, 0 \leq s \leq \tau_{y}^{b}, X_{s \vee \tau_{y}^{b}}-2 r Y_{s \vee \tau_{y}^{b}} \leq a-2 r b, \tau_{y}^{b} \leq s \leq t, Y_{t \vee \tau_{y}^{b}} \geq b\right) \\
& =\mathbb{E}\left[\mathbf{1}_{\left\{\max _{s \in\left[0, \tau_{y}^{b}\right]} X_{s}<a\right\}} \mathbb{E}\left[\max _{s \in\left[\tau_{y}^{b}, t\right]}\left(X_{s}-2 r Y_{s}\right) \leq a-2 r b, Y_{t} \geq b, \tau_{y}^{b} \leq t \mid \mathcal{F}_{\tau_{y}^{b}}\right]\right] \\
& =\mathbb{E}\left[\mathbf{1}_{\left\{\max _{s \in\left[0, \tau_{y}^{b}\right]} X_{s}<a\right\}} \mathbb{E}\left[\max _{s \in\left[\tau_{y}^{b}, t\right]}\left(X_{s}-2 r Y_{s}\right) \leq a-2 r b, Y_{t} \geq b, \tau_{y}^{b} \leq t \mid\left(X_{\tau_{y}^{b}}, Y_{\tau_{y}^{b}}\right)\right]\right] \text {. }
\end{aligned}
$$


The conditional expectation in the above integral can be expressed in the form $g\left(X_{\tau_{y}^{b}}\right)$ for some integrable function since $Y_{\tau_{y}^{b}} \equiv b$. For simplicity of notation, in the following we will use $g$ to replace the conditional expectation. Then

$$
\mathbb{P}\left(\tau_{x}^{a}>t, \tau_{y}^{b} \leq t, Y_{t} \leq b\right)=\mathbb{E}\left[g\left(X_{\tau_{y}^{b}}\right) \mathbf{1}_{\left\{\tau_{y}^{b} \leq t, \tau_{y}^{b}<\tau_{x}^{a}\right\}}\right]
$$

In (2.3), replacing $\mathbf{1}_{\left\{\tau_{y}^{b}<\tau_{x}^{a}\right\}}$ by 1 , we obtain

$$
\mathbb{P}\left(\tau_{x}^{a}>t, \tau_{y}^{b} \leq t, Y_{t} \leq b\right) \leq \mathbb{E}\left[g\left(X_{\tau_{y}^{b}}\right) \mathbf{1}_{\left\{\tau_{y}^{b} \leq t\right\}}\right] .
$$

Since $\left(X_{t}\right)_{t \geq 0}$ and $\left(Y_{t}\right)_{t \geq 0}$ are Brownian motions with normal joint distribution and constant correlation coefficient, there exists a Brownian motion $\left(B_{t}\right)_{t \geq 0}$ independent of $\left(Y_{t}\right)_{t \geq 0}$ such that

$$
X_{t}=\sqrt{1-r^{2}} B_{t}+r Y_{t} .
$$

In fact, $B_{t}$ could be defined as $\left(X_{t}-r Y_{t}\right) / \sqrt{1-r^{2}}$. Then we can obtain the joint distribution of $X_{\tau_{y}^{b}}$ and $\tau_{y}^{b}$. Therefore,

$$
\begin{aligned}
\mathbb{E}\left[g\left(X_{\tau_{y}^{b}}\right) \mathbf{1}_{\left\{\tau_{y} \leq t\right\}}\right]= & \int_{0}^{t}\left(\int_{-\infty}^{+\infty} g\left(\sqrt{1-r^{2}} x+r b\right) \frac{1}{\sqrt{2 \pi u}} \mathrm{e}^{-x^{2} / 2 u} \mathrm{~d} x\right) \mathbb{P}_{\tau_{y}^{b}}(\mathrm{~d} u) \\
= & \int_{0}^{t}\left(\int_{-\infty}^{+\infty} g(x) \frac{1}{\sqrt{2 \pi u\left(1-r^{2}\right)}} \mathrm{e}^{-(x-r b)^{2} / 2 u\left(1-r^{2}\right)} \mathrm{d} x\right) \\
& \times \frac{b}{\sqrt{2 \pi u^{3}}} \mathrm{e}^{-b^{2} / 2 u} \mathrm{~d} u,
\end{aligned}
$$

where $\mathbb{P}_{\tau_{y}^{b}}$ stands for the distribution functions of $\tau_{y}^{b}$, which can be calculated via (1.1).

Now we calculate $g\left(X_{\tau_{y}^{b}}\right)$. When $\tau_{y}^{b}=u$ and $X_{\tau_{y}^{b}}=x$, then, by virtue of Lemma 2.1,

$$
\begin{aligned}
g(x) & =\mathbb{E}\left[\max _{s \in[u, t]}\left(X_{s}-2 r Y_{s}\right) \leq a-2 r b, Y_{t} \geq b \mid\left(X_{u}=x, Y_{u}=b\right)\right] \\
& =\mathbb{P}\left(\max _{s \in[0, t-u]}\left(X_{s}-2 r Y_{s}\right) \leq a-x, Y_{t-u} \geq 0\right) \\
& =\mathbb{P}\left(\max _{s \in[0, t-u]} X_{s} \leq a-x, Y_{t-u} \leq 0\right) .
\end{aligned}
$$

When $x \geq a$, we know that $\mathbb{P}\left(\max _{s \in[0, t-u]} X_{s} \leq a-x\right)$ is equal to 0 . So, in the following, we assume that $x<a$. Set $\tau_{x}^{a-x}=\inf \left\{s \geq 0 ; X_{s} \geq a-x\right\}$. The last term in the above equation can be calculated using Lemma 2.2. More precisely,

$$
\begin{aligned}
& \mathbb{P}\left(\max _{s \in[0, t-u]} X_{s} \leq a-x, Y_{t-u} \geq 0\right) \\
&=\mathbb{P}\left(\tau_{x}^{a-x} \geq t-u, Y_{t-u} \leq 0\right) \\
&=\mathbb{P}\left(Y_{t-u} \leq 0\right)-\mathbb{P}\left(Y_{t-u} \leq 0, \tau_{x}^{a-x}<t-u, X_{t-u} \geq a-x\right) \\
& \quad-\mathbb{P}\left(Y_{t-u} \leq 0, \tau_{x}^{a-x}<t-u, X_{t-u}<a-x\right) \\
&=\mathbb{P}\left(Y_{t-u} \leq 0\right)-\mathbb{P}\left(Y_{t-u} \leq 0, X_{t-u} \geq a-x\right) \\
&-\mathbb{P}\left(Y_{t-u}-2 r X_{t-u} \leq-2 r(a-x), X_{t-u}>a-x\right) \\
&= \mathbb{P}\left(Y_{t-u} \leq 0, X_{t-u}<a-x\right)-\mathbb{P}\left(Y_{t-u} \leq-2 r(a-x), X_{t-u}<x-a\right) .
\end{aligned}
$$


Finally, when $\tau_{y}^{b}=u \leq t$ and $X_{\tau_{y}^{b}}=x$, we obtain

$$
g(x)= \begin{cases}\mathbb{P}\left(Y_{t-u} \leq 0, X_{t-u}<a-x\right)-\mathbb{P}\left(Y_{t-u} \leq 2 r(x-a), X_{t-u}<x-a\right), & x<a, \\ 0, & x \geq a\end{cases}
$$

Altogether, we obtain

$$
\begin{gathered}
\mathbb{P}\left(\tau_{x}^{a}>t, \tau_{y}^{b} \leq t, Y_{t} \leq b\right) \\
\leq \int_{0}^{t}\left(\int_{-\infty}^{a}\left[\mathbb{P}\left(X_{t-u}<a-x, Y_{t-u} \leq 0\right)-\mathbb{P}\left(X_{t-u}<x-a, Y_{t-u} \leq-2 r(a-x)\right)\right]\right. \\
\left.\quad \times \frac{1}{\sqrt{2 \pi u\left(1-r^{2}\right)}} \mathrm{e}^{-(x-r b)^{2} / 2 u\left(1-r^{2}\right)} \mathrm{d} x\right) \frac{b}{\sqrt{2 \pi u^{3}}} \mathrm{e}^{-b^{2} / 2 u} \mathrm{~d} u,
\end{gathered}
$$

which yields the desired result upon applying the joint distribution of $X_{t-u}$ and $Y_{t-u}$.

Remark 2.1. In the proof of Lemma 2.4, by making use of a formula given in [6, Equation (15)] and [7, Equations (1.5) and (1.6)], we can calculate $\mathbb{E}\left[g\left(X_{\tau_{y}^{b}}\right) \mathbf{1}_{\left\{\tau_{y}^{b}<t, \tau_{y}^{b}<\tau_{x}^{a}\right\}}\right]$. More precisely, setting $\tau=\tau_{x}^{a} \wedge \tau_{y}^{b}$,

$$
\begin{aligned}
\mathbb{P}\left(X(\tau) \in \mathrm{d} x, \tau=\tau_{y}^{b} \in \mathrm{d} t\right)= & \frac{\pi}{\alpha^{2} t(a-x)} \exp \left[-\frac{1}{2 t}\left(\frac{(a-x)^{2}}{1-r^{2}}+r_{0}^{2}\right)\right] \\
& \times \sum_{n=1}^{\infty} n \sin \left(\frac{n \pi \theta_{0}}{\alpha}\right) I_{n \pi / \alpha}\left(\frac{a-x}{\sqrt{1-r^{2}}} \frac{r_{0}}{t}\right) \mathrm{d} x \mathrm{~d} t
\end{aligned}
$$

where $x$ varies from $-\infty$ to $a,\left(r_{0}, \theta_{0}\right)$ denotes the polar coordinates of the point $((a-$ $\left.r b) / \sqrt{1-r^{2}}, b\right)$, and $\alpha$ is defined in (1.4). Therefore,

$$
\begin{aligned}
\mathbb{E}\left[g\left(X_{\tau_{y}^{b}}\right) \mathbf{1}_{\left\{\tau_{y}^{b}<t, \tau_{y}^{b}<\tau_{x}^{a}\right\}}\right]= & \int_{0}^{t} \int_{-\infty}^{a} g(x) \frac{\pi}{\alpha^{2} t(a-x)} \exp \left[-\frac{1}{2 t}\left(\frac{(a-x)^{2}}{1-r^{2}}+r_{0}^{2}\right)\right] \\
& \times \sum_{n=1}^{\infty} n \sin \left(\frac{n \pi \theta_{0}}{\alpha}\right) I_{n \pi / \alpha}\left(\frac{r_{0}(a-x)}{t \sqrt{1-r^{2}}}\right) \mathrm{d} x \mathrm{~d} t .
\end{aligned}
$$

This is an equality for the probability $\mathbb{P}\left(\tau_{x}^{a}>t, \tau_{y}^{b} \leq t, Y_{t} \leq b\right)$. It does not omit any information. However, we do not make use of this equality, since it is very difficult to deal with the double integral over the Bessel functions.

Proof of Corollary 1.1. It is enough to prove this corollary for $t=1$. Since $\mathbb{P}\left(Y_{1}^{*} \geq b\right)=$ $2 \Phi(-b)$, and, by Theorem 1.1, $\mathbb{P}\left(X_{1}^{*} \geq b, Y_{1}^{*} \geq b\right) \leq U_{1}(b, b)$ for $t=1$, we have

$$
\begin{aligned}
\limsup _{b \rightarrow \infty} & \frac{\mathbb{P}\left(X_{1}^{*} \geq b, Y_{1}^{*} \geq b\right)}{\mathbb{P}\left(Y_{1}^{*} \geq b\right)} \\
\leq & \frac{1}{2}+\lim _{b \rightarrow \infty} \frac{1}{2 \Phi(-b)}\left[\int_{-\infty}^{-b}\left(\int_{(1-2 r) b}^{\infty} \varphi(x, y) \mathrm{d} y\right) \mathrm{d} x+\int_{b}^{\infty}\left(\int_{b}^{\infty} \varphi(x, y) \mathrm{d} y\right) \mathrm{d} x\right] .
\end{aligned}
$$


Using l'Hôpital's rule,

$$
\begin{aligned}
\lim _{b \rightarrow \infty} & \frac{1}{2 \Phi(-b)} \int_{b}^{\infty} \int_{b}^{\infty} \varphi(x, y) \mathrm{d} y \mathrm{~d} x \\
& =\lim _{b \rightarrow \infty} \frac{1}{\sqrt{2 \pi\left(1-r^{2}\right)}} \mathrm{e}^{b^{2} / 2} \int_{b}^{\infty} \exp \left[-\frac{x^{2}-2 r b x+b^{2}}{2\left(1-r^{2}\right)}\right] \mathrm{d} x \\
& =\lim _{b \rightarrow \infty} \frac{1}{\sqrt{2 \pi\left(1-r^{2}\right)}} \int_{(1-r) b}^{\infty} \mathrm{e}^{-x^{2} / 2\left(1-r^{2}\right)} \mathrm{d} x \\
& =0
\end{aligned}
$$

Similarly,

$$
\begin{aligned}
\lim _{b \rightarrow \infty} & \frac{1}{2 \Phi(-b)} \int_{-\infty}^{-b}\left(\int_{(1-2 r) b}^{\infty} \varphi(x, y) \mathrm{d} y\right) \mathrm{d} x \\
= & \lim _{b \rightarrow \infty} \frac{\mathrm{e}^{b^{2} / 2}}{2 \sqrt{2 \pi\left(1-r^{2}\right)}} \\
& \times\left[\int_{(1-2 r) b}^{\infty} \mathrm{e}^{-\left(b^{2}+2 r b y+y^{2}\right) / 2\left(1-r^{2}\right)} \mathrm{d} y\right. \\
& \left.\quad+(1-2 r) \int_{b}^{\infty} \exp \left[-\frac{x^{2}+2 r(1-2 r) b x+(1-2 r)^{2} b^{2}}{2\left(1-r^{2}\right)}\right] \mathrm{d} x\right] \\
= & \frac{1}{2 \sqrt{2 \pi\left(1-r^{2}\right)}}\left(\lim _{b \rightarrow \infty} I+\lim _{b \rightarrow \infty} I I\right) .
\end{aligned}
$$

For the first part,

$$
\lim _{b \rightarrow \infty} I=\lim _{b \rightarrow \infty} \int_{(1-2 r) b}^{\infty} \mathrm{e}^{-(y+r b)^{2} / 2\left(1-r^{2}\right)} \mathrm{d} y=\lim _{b \rightarrow \infty} \int_{(1-r) b}^{\infty} \mathrm{e}^{-y^{2} / 2\left(1-r^{2}\right)} \mathrm{d} y=0 .
$$

For the second part,

$$
\begin{aligned}
\lim _{b \rightarrow \infty} I I & =\lim _{b \rightarrow \infty}(1-2 r) \int_{b}^{\infty} \exp \left[-\frac{(x+r(1-2 r) b)^{2}}{2\left(1-r^{2}\right)}\right] \exp \left[-\frac{\left(1-4 r-4 r^{2}\right) b^{2}-b^{2}}{2}\right] \mathrm{d} x \\
& =(1-2 r) \lim _{b \rightarrow \infty} \mathrm{e}^{2 r(1-r) b^{2}} \int_{\left(1+r-2 r^{2}\right) b}^{\infty} \mathrm{e}^{-x^{2} / 2\left(1-r^{2}\right)} \mathrm{d} x .
\end{aligned}
$$

When $r \in(-1,0), \mathrm{e}^{2 r(1-r) b^{2}}$ tends to 0 , and

$$
\int_{\left(1+r-2 r^{2}\right) b}^{\infty} \mathrm{e}^{-x^{2} / 2\left(1-r^{2}\right)} \mathrm{d} x<\int_{-\infty}^{\infty} \mathrm{e}^{-x^{2} / 2\left(1-r^{2}\right)} \mathrm{d} x
$$

is finite, so $\lim _{b \rightarrow \infty} I I=0$ when $r \in(-1,0]$. When $r \in(0,1)$,

$$
\begin{aligned}
\lim _{b \rightarrow \infty} \mathrm{e}^{2 r(1-r) b^{2}} \int_{\left(1+r-2 r^{2}\right) b}^{\infty} \mathrm{e}^{-x^{2} / 2\left(1-r^{2}\right)} \mathrm{d} x \\
\quad=\lim _{b \rightarrow \infty} \frac{\left(1+r-2 r^{2}\right) \mathrm{e}^{-\left(1+r-2 r^{2}\right)^{2} b^{2} / 2\left(1-r^{2}\right)}}{4 r(1-r) b \mathrm{e}^{-2 r(1-r) b^{2}}} \\
\quad=\lim _{b \rightarrow \infty} \frac{2 r+1}{4 r b} \exp \left[-\frac{(1-r)\left(2 r^{2}+2 r+1\right)}{2(1+r)} b^{2}\right] \\
=0 \quad \text { as } r \in(0,1) .
\end{aligned}
$$


Hence,

$$
\lim _{b \rightarrow \infty} \frac{1}{2 \Phi(-b)} \int_{-\infty}^{-b}\left(\int_{(1-2 r) b}^{\infty} \varphi(x, y) \mathrm{d} y\right) \mathrm{d} x=0 .
$$

Altogether, we obtain

$$
\limsup _{b \rightarrow \infty} \frac{\mathbb{P}\left(X_{1}^{*} \geq b, Y_{1}^{*} \geq b\right)}{\mathbb{P}\left(Y_{1}^{*} \geq b\right)} \leq \frac{1}{2} \quad \text { for } r \in(-1,1),
$$

completing the proof.

\section{Proof of Theorem 1.2}

Let $\left(X_{t}\right)_{t \geq 0}$ and $\left(Y_{t}\right)_{t \geq 0}$ be two $\mathcal{F}_{t}$-Brownian motions with normal joint distribution. Assume that hypotheses (H1) and (H2) hold. For any positive constant $a, b, c$, and $d$, in order to estimate the crossing probability

$$
\mathbb{P}\left(X_{t} \leq a t+b, Y_{t} \leq c t+d, 0 \leq t \leq T\right),
$$

where $T$ is a positive constant, we first establish a kind of Girsanov theorem, then use Theorem 1.1 to deduce its lower and upper estimates. Novikov [8] used this idea to estimate the exit probability of one-dimensional Brownian motion to a moving boundary.

Proposition 3.1. (Girsanov's theorem.) Let

$$
W\left(\mathbb{R}^{2}\right)=\left\{x:[0, T] \rightarrow \mathbb{R}^{2} \text { continuous } ; X(0)=0\right\} .
$$

For any constants $a$ and $b$, let $\eta_{a, b}: W\left(\mathbb{R}^{2}\right) \rightarrow W\left(\mathbb{R}^{2}\right)$ be defined as

$$
t \mapsto \eta_{a, b}(x(\cdot), y(\cdot))(t)=(x(t)+a t, y(t)+b t)
$$

for $t \in[0, T]$. Denote by $\mu_{\eta}$ the induced measure of $\mu_{0}$ by the map $\eta_{a, b}$, i.e. $\mu_{\eta}=\mu_{0} \circ \eta^{-1}$. Then $\mu_{\eta}$ is absolutely continuous with respect to $\mu_{0}$ and

$$
\frac{\mathrm{d} \mu_{\eta}}{\mathrm{d} \mu_{0}}(x(\cdot), y(\cdot))=\exp \left[\frac{1}{2\left(1-r^{2}\right)}\left\{2(a-r b) x(T)+2(b-r a) y(T)-\left(a^{2}+b^{2}-2 r a b\right) T\right\}\right] .
$$

Proof. The proof is similar to that of Girsanov's theorem in the one-dimensional case. We omit the details, and refer the reader to [5, Chapter 7, Theorem 3.1] for more general results in this case.

Proof of Theorem 1.2. We first consider the upper bound. By Proposition 3.1 and Hölder's inequality, we obtain

$$
\begin{aligned}
\mathbb{P}\left(X_{t}\right. & \left.\leq a t+c, Y_{t} \leq b t+d, 0 \leq t \leq T\right) \\
& =\mathbb{P}\left(X_{t}-a t \leq c, Y_{t}-b t \leq d, 0 \leq t \leq T\right) \\
& =\mathbb{E}\left[\mathbf{1}_{\left\{X_{t} \leq c, Y_{t} \leq d, 0 \leq t \leq T\right\}} \tilde{M}_{T}\right] \\
& \leq \mathbb{P}\left(X_{t} \leq c, Y_{t} \leq d, 0 \leq t \leq T\right)^{1 / p}\left(\mathbb{E} \tilde{M}_{T}^{p /(p-1)}\right)^{(p-1) / p}
\end{aligned}
$$

which gives the upper estimate. 
On the other hand,

$$
\begin{aligned}
\mathbb{P}\left(X_{t}\right. & \left.\leq c, Y_{t} \leq d, 0 \leq t \leq T\right) \\
& =\mathbb{P}\left(X_{t}+a t \leq a t+c, Y_{t}+b t \leq b t+d, 0 \leq t \leq T\right) \\
& =\mathbb{E}\left[\mathbf{1}_{\left\{X_{t} \leq a t+c, Y_{t} \leq b t+d, 0 \leq t \leq T\right\}} M_{T}\right] \\
& \leq \mathbb{P}\left(X_{t} \leq a t+c, Y_{t} \leq b t+d, 0 \leq t \leq T\right)^{1 / p}\left(\mathbb{E} M_{T}^{p /(p-1)}\right)^{(p-1) / p} .
\end{aligned}
$$

This yields

$$
\begin{aligned}
\mathbb{P}\left(X_{t}\right. & \left.\leq a t+c, Y_{t} \leq b t+d, 0 \leq t \leq T\right) \\
& \geq \mathbb{P}\left(X_{t} \leq c, Y_{t} \leq d, 0 \leq t \leq T\right)^{p}\left(\mathbb{E} M_{T}^{p /(p-1)}\right)^{1-p},
\end{aligned}
$$

which gives the lower bound.

\section{Acknowledgements}

This work was supported by FANEDD (number 200917) and Project 985. The first author is very grateful to Prof. Liqun Wang for valuable discussions on this topic and for providing his lecture notes. The authors would like to thank the anonymous referee for valuable suggestions which helped improve the present paper.

\section{References}

[1] Anderson, T. W. (1960). A modification of the sequential probability ratio test to reduce the sample size. Ann. Math. Statist. 31, 165-197.

[2] Doob, J. L. (1949). Heuristic approach to the Kolmogorov-Smirnov theorems. Ann. Math. Statist. 20, $393-403$.

[3] Durbin, J. (1992). The first-passage density of the Brownian motion process to a curved boundary. J. Appl. Prob. 29, 291-304.

[4] Feller, W. (1966). An introduction to Probability Theory and Its Applications, Vol. 2. John Wiley, New York.

[5] Friedman, A. (2006). Stochastic Differential Equations and Applications. Dover Publications, Mineola, NY.

[6] IYengar, S. (1985). Hitting lines with two-dimensional Brownian motion. SIAM J. Appl. Math. 45, $983-989$.

[7] Metzler, A. (2010). On the first passage problem for correlated Brownian motion. Statist. Prob. Lett. 80, 277-284.

[8] Novikov, A. A. (1981). On estimates and the asymptotic behavior of nonexit probabilities of a Wiener process to a moving boundary. Math. USSR SB 38, 495-505.

[9] Ricciardi, L. M., SACERdote, L. AND SAto, S. (1984). On an integral equation for first-passage-time probability densities. J. Appl. Prob. 21, 302-314.

[10] Siegmund, D. (1986). Boundary crossing probabilities and statistical applications. Ann. Math. Statist. 14, 361404.

[11] Wang, L. ANd Pötzelberger, K. (1997). Boundary crossing probability for Brownian motion and general boundaries. J. Appl. Prob. 34, 54-65.

[12] Pötzelberger, K. And Wang, L. (2001). Boundary crossing probability for Brownian motion. J. Appl. Prob. 38, $152-164$. 